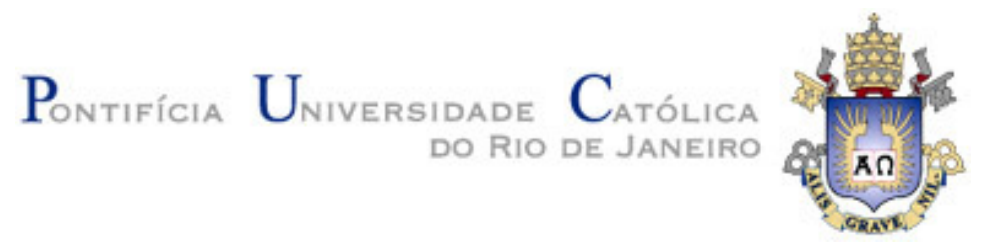

Ismael Humberto Ferreira dos Santos

\title{
A Collaborative Environment for Offshore Engineering Simulations based on Visualization and Workflow
}

TESE DE DOUTORADO

Thesis presented to the Postgraduate Program in Informatics of the Departamento de Informática, PUC-Rio as partial fulfillment of the requirements for the degree of Doutor em Informática.

Advisor: Prof. Marcelo Gattass Co-advisor: Prof. Alberto Barbosa Raposo

Rio de Janeiro

April 2010 


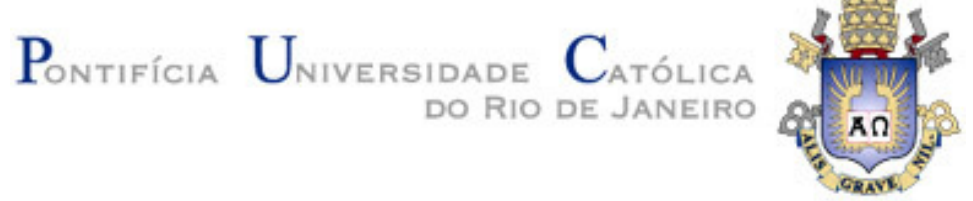

Ismael Humberto Ferreira dos Santos

\title{
A Collaborative Environment for Offshore Engineering Simulations based on Visualization and Workflow
}

\begin{abstract}
Thesis presented to the Postgraduate Program in Informatics, of the Departamento de Informática do Centro Técnico Científico da PUC-Rio, as partial fulfillment of the requirements for the degree of Doutor.
\end{abstract}

\author{
Prof. Marcelo Gattass \\ Advisor \\ Departamento de Informática - PUC-Rio \\ Prof. Alberto Barbosa Raposo \\ Co-Advisor \\ Departamento de Informática - PUC-Rio \\ Prof. Bruno Feijó \\ Departamento de Informática - PUC-Rio \\ Prof. Marco Antonio Casanova \\ Departamento de Informática - PUC-Rio
}

Profa. Judith Kelner Centro de Informática - UFPE

Profa. Marta Lima de Queirós Mattoso Departamento Engenharia de Sistemas e Computação - COPPE-UFRJ

Profa. Veronica Teichrieb Centro de Informática - UFPE

Prof. José Eugenio Leal Coordinator of the Centro Técnico Científico - PUC-Rio 
All rights reserved.

\section{Ismael Humberto Ferreira dos Santos}

Graduated in Applied Mathematics from Universidade Federal do Rio de Janeiro - UFRJ in 1997. He also has a master's degree in Applied Mathematics from the Federal University of Rio de Janeiro. He works for Petrobras (Brazilian Oil company) since 1987.

Bibliographic data

Santos, Ismael Humberto Ferreira dos

A Collaborative Environment for Offshore Engineering Simulations based on Visualization and Workflow / Ismael Humberto Ferreira dos Santos; advisor: Marcelo Gattass ; co-advisor: Alberto Barbosa Raposo. - 2010.

145 f. : il. (color.) ; $30 \mathrm{~cm}$

Tese (doutorado)-Pontifícia Universidade Católica do Rio de Janeiro, Departamento de Informática, 2010.

Inclui bibliografia

1. Informática - Teses. 2. Trabalho colaborativo auxiliado por computador. 3. Ambientes colaborativos para a solução de problemas. 4. Sistemas gerenciadores de experimentos científicos (workflows científicos). 5. Visualização colaborativa. 6. Ambientes virtuais colaborativos. 7. Engenharia offhore. 8. Óleo e gás. I. Gattass, Marcelo. II. Raposo, Alberto Barbosa. III. Pontifícia Universidade Católica do Rio de Janeiro. Departamento de Informática. IV. Título. 
Aos meus pais, Ubirajara e Dalva, aos meus queridos filhos João Guilherme e Pedro Henrique e a minha amada esposa Yone. 


\section{Acknowledgments}

A meus pais, Ubirajara e Dalva, por tudo que sou hoje.

A minha esposa, Yone, pelo seu amor e incentivo, e pela abnegação com que abdicou de muitas comodidades e assumiu tarefas que me cabiam.

Aos meus filhos, João Guilherme e Pedro Henrique, que são a razão da minha existência.

Aos meus orientadores Marcelo Gattass e Alberto Barbosa Raposo, pelo invulgar empenho que dedicaram a esta empreitada, pelos conselhos oportunos em momentos decisivos da consecução da pesquisa e pela confiança que depositaram em mim desde o início.

Ao meu ex-Gerente e amigo Álvaro Maia pela oportunidade que me deu confiando na minha capacidade de realização desse doutorado.

Ao meu Gerente e também amigo Augustu Petrus Levy por depositar uma confiança infindável no meu trabalho.

A Petróleo Brasileiro S. A. e ao CENPES, por todo o apoio e suporte financeiro que foram fundamentais por terem proporcionado a oportunidade e os meios para realizar esta pesquisa.

Aos amigos da Petrobrás, Heitor Araújo, Luciano Pereira dos Reis, Rodrigo Toledo pelo incentivo e motivação para continuar nessa jornada.

Aos meus amigos Rogério Pinheiro, Paulo Gallotti e a equipe do Environ, que tanto me ajudaram na implementação desta tese.

Aos amigos do TecGraf e da Puc-Rio, por terem proporcionado não só um ambiente propício para a pesquisa mas também um ambiente de extrema camaradagem e companheirismo.

Aos componentes da banca, por suas sugestões. 


\section{Abstract}

Santos, Ismael Humberto Ferreira dos; Gattass, Marcelo; Raposo, Alberto Barbosa. A Collaborative Environment for Offshore Engineering Simulations based on Visualization and Workflow. Rio de Janeiro, 2010. 145p. DSc. Thesis - Departamento de Informática, Pontifícia Universidade Católica do Rio de Janeiro.

Deep-water production systems, including floating production units (platforms or ships) and all the equipments playing a part in the production process, are currently designed by means of complex computational modeling systems. Those systems involve the areas of structural calculus, meteo-oceanography (currents, waves and wind forces), hydrodynamics, risers (rigid or flexible steel pipes for carrying oil from the well in subsurface up to the production unit), mooring systems, submarine equipment, seabed foundations and Geologic/Geotechnical risk assessment. The project of a new production unit is a lengthy and expensive process, that can last many years and consume hundreds of million of dollars, depending on the complexity of the unit and how mature is the technology developed to make the project technically and economically feasible. Projects are conducted by diverse specialists, sometimes geographically distributed, yielding independent but highly interrelated artifacts and results. The need for collaboration is an inherent characteristic of deep-water floating production unit projects. The possibility to share information among users, control the execution of different modeling tools, visualize and manipulate virtual 3D models in immersive Virtual Reality (VR) environments is pushing the limits of teamwork activities in oil \& gas industry especially in Offshore Engineering. The objective of this thesis is to establish the fundamental principles and address the main issues in the development of a Collaborative Environment for Engineering, named CEE (Collaborative Engineering Environment), in order to allow the collaborative visualization and interpretation of simulation results produced in engineering projects, which in general also involve different specialties. Due to the multidisciplinary characteristic of those projects, collaborative visualization becomes a key component during the life cycle of engineering projects, especially those in Offshore Engineering, used in this work as case of study. We propose an 
integrated collaborative environment to be used by project engineers' teams during the execution and control of complex engineering projects, as is the case of the projects of deep-water floating production units. The system requirements were carefully compiled aiming to enable an effective collaboration among the participants, creating a suitable environment for discussing, validating, interpreting and documenting the results of the simulations executed during the different phases of an engineering project. To further improve the interpretation capacity and a better comprehension of results the support for immersive 3D visualization is also available in the visualization tool, especially tailored for the Offshore Engineering domain. In order to meet these goals, we devise a ServiceOriented Architecture (SOA) for CEE. This architecture is composed of the integration of different technologies of Computer Supported Collaborative Work (CSCW), Virtual Reality (VR) and Grid Computing (GC). We use a Scientific Workflow Management System (ScWfMS), based on BPEL (Business Process Execution Language), a Grid-enabled software infrastructure for executing engineering simulations, and a Video Conferencing system (VCS) to furnish audio and video collaboration. For visualizing the results, a VR visualization tool, specialized for Offshore Engineering, ENVIRON, has also been developed in conjunction with the PUC-Rio/TecGraf team.

\section{Keywords}

Computer-Supported Cooperative Work; Scientific Workflow Management Systems; Collaborative Problem Solving Environments; Collaborative Visualization; Collaborative Virtual Environments; Offshore Engineering; Oil \& Gas. 


\section{Resumo}

Santos, Ismael Humberto Ferreira dos; Gattass, Marcelo; Raposo, Alberto Barbosa. Um Ambiente Colaborativo para Simulações em Engenharia Offshore baseado em Visualização e Workflow. Rio de Janeiro, 2010. 145p. Tese de Doutorado - Departamento de Informática, Pontifícia Universidade Católica do Rio de Janeiro.

Os sistemas de produção de petróleo em águas profundas, incluindo as unidades flutuantes de produção (plataformas ou navios) e todos os equipamentos que participam da produção são atualmente projetados por complexos sistemas de modelagem computacional. Tais sistemas envolvem as áreas de cálculo estrutural, meteo-oceanografia (forças de correntes, ondas e ventos), hidrodinâmica, risers (tubos de aço rígidos ou flexíveis para levar o óleo do poço em sub-superfície até a unidade de produção), sistemas de ancoragem, equipamentos submarinos, fundações e avaliação de risco geológico-geotécnico. O projeto de uma nova unidade de produção é um processo longo e custoso, podendo durar anos e consumir centenas de milhões de dólares, dependendo da complexidade da unidade e da maturidade da tecnologia desenvolvida para tornar o projeto econômica e tecnicamente viável. Os projetos são conduzidos por diversos especialistas, por vezes geograficamente dispersos, gerando artefatos e resultados independentes, porém altamente inter-relacionados. A necessidade de colaboração é uma característica inerente aos projetos de unidades flutuantes de produção para águas profundas. A possibilidade de compartilhar informações entre usuários, controlar a execução de diferentes ferramentas de modelagem, visualizar e manipular modelos 3D virtuais em ambientes imersivos de Realidade Virtual vem empurrando os limites das atividades dos times na indústria do petróleo especialmente em Engenharia de Petróleo. O objetivo desta tese é o de fundamentar os princípios e equacionar os principais problemas para o desenvolvimento de um Ambiente Colaborativo para Engenharia, denominado CEE (Collaborative Engineering Environment), de forma a permitir a visualização colaborativa e interpretação dos resultados de simulações criadas nos projetos de engenharia, que em geral envolvem também 
diferentes especialidades. Devido à característica multidisciplinar dos projetos, a visualização colaborativa torna-se um componente de fundamental importância durante o ciclo de vida de projetos de engenharia, especialmente os da área de Engenharia Offshore, utilizada neste trabalho como caso de estudo. Propomos um ambiente integrado para visualização colaborativa a ser usado pelas equipes de engenheiros projetistas durante a execução e controle de projetos de engenharia complexos como é o caso dos projetos de unidades flutuantes de produção para águas profundas. Os requisitos do sistema foram levantados com o objetivo de permitir uma colaboração efetiva entre os participantes, criando um ambiente propício para discussão, validação, interpretação e documentação dos resultados das simulações executadas durante as fases de um projeto de engenharia. Para aumentar ainda mais a capacidade de interpretação e uma melhor compreensão dos resultados o suporte a visualização em ambientes imersivos 3D também esta disponibilizado na ferramenta de visualização utilizada, que foi especialmente adaptada para a área de Engenharia Offhore.

Para atingir estes objetivos, propomos uma Arquitetura Orientada a Serviços para o CEE. Esta arquitetura é composta pela integração de diferentes tecnologias de Trabalho Colaborativo Auxiliado por Computador (CSCW), Realidade Virtual e Computação em Grade. Utiliza-se um sistema de Gerência de Workflows de Experimentos Científicos (ScWfMS), baseado em BPEL (Business Process Execution Language), para execução de simulações de engenharia em uma infra-estrutura de computação em grade subjacente e um sistema de Videoconferência (VCS) para suporte a colaboração de áudio e vídeo. Para a visualização dos resultados um sistema de visualização, especializado para Engenharia Offshore, ENVIRON, foi desenvolvido em conjunto com a equipe da PUC-Rio/TecGraf.

\section{Palavras-chave}

Trabalho Colaborativo Auxiliado por Computador; Ambientes Colaborativos para a Solução de Problemas; Sistemas Gerenciadores de Experimentos Científicos (Workflows Científicos); Visualização Colaborativa; Ambientes Virtuais Colaborativos; Engenharia Offhore; Óleo \& Gas. 


\section{Summary}

1 Introduction 19

1.1 Motivation: Large Scale Engineering Projects (LSEP) 19

1.1.1 The Role of Visualization, Remote Collaboration and High Performance Computing $\quad 20$

1.2 Offshore Engineering Projects 23

$\begin{array}{lll}1.3 & \text { Problem Solving Environments } & 26\end{array}$

1.3.1 Collaborative Problem Solving Environments 27

1.4 CEE - Collaborative Problem Solving Environment for Offshore Engineering 28

1.5 Thesis Main Contributions 30

$\begin{array}{lll}1.6 & \text { Thesis Outline } & 30\end{array}$

2 Related Work 32

2.1 Workflow Management Systems and Grid Computing 32

2.1.1 Data Driven Multiphysics Simulation Framework (DDMSF) 33

2.1.2 Wind Tunnel 35

\begin{tabular}{ll}
2.1 .3 & Vistrails \\
\hline
\end{tabular}

$\begin{array}{lll}2.1 .4 & \text { Discussion } & 38\end{array}$

2.2 Virtual Reality Visualization Technology 39

2.2.1 Immersive Well Path Planning 40

2.2.2 VRGeo Demonstrator 40

2.2.3 Geological-Mapping and Displacement Analysis (GMDA) 42

2.2.4 Discussion 43

2.3 Service-Oriented Architecture 43

2.3.1 Real-Time Architecture Project (RTAP) 44

2.3.2 Integrated Asset Management framework (IAM) 45

$\begin{array}{lll}\text { 2.3.3 Discussion } & 47\end{array}$

$\begin{array}{lll}2.4 & \text { CEE Main Ideas } & 47\end{array}$

3 CEE Conceptual Model 50

3.1 Collaborative Visualization Environment 52

3.1.1 Video Conferencing $\quad 55$ 
3.1.2 VR Visualization $\quad 56$

3.1.3 Collaboration Manager and Collaboration Bus 57

3.2 Scientific Workflow Environment 59

3.2.1 Scientific Workflow Service 60

3.2.2 Data Access and Engineering Simulations Execution Interoperability 62

3.2.3 Grid Computing Infrastructure (GCl) 63

3.3 CEE SOA Architecture 63

$\begin{array}{lll}3.4 & \text { CEE Usage Scenario Overview } & 65\end{array}$

4 CEE SOA Architecture $\quad 70$

4.1 CEE Architecture Layers 70

4.1.1 Technology Layer $\quad 70$

4.1.2 Collaborative Engineering Layer 72

4.2 Application Layer $\quad 72$

4.2.1 CEE Client Services $\quad 74$

4.2.2 CEE Server Services and Components 74

4.2.3 Registration of Services 80

4.2.4 Project Workflow Service $\quad 81$

4.2.5 Scientific Workflow Service 82

4.2.6 Videoconference Service $\quad 84$

$\begin{array}{lll}\text { 4.2.7 Collaborative Visualization Service } & 87\end{array}$

5 CEE Application Scenarios $\quad 92$

5.1 Collaborative Risers Analysis Workflow 92

5.1.1 BPEL Scientific Workflow 92

5.1.2 Video Conferencing 95

5.1.3 3D Annotations 96

$\begin{array}{lll}5.1 .4 & \text { 3D Measurements } & 98\end{array}$

5.2 Design Review Workflow 101

5.2.1 Virtual Guided Tour 104

6 Conclusions and Future Work 106

$\begin{array}{lll}6.1 & \text { Conclusions } & 106\end{array}$

$\begin{array}{ll}6.2 & \text { Future Work } \\ & 108\end{array}$

$\begin{array}{lll}\text { 6.2.1 Project Workflow Environment } & 108\end{array}$

$\begin{array}{ll}\text { 6.2.2 Scientific Workflow Environment } & 108\end{array}$

6.2.3 Collaborative Visualization Environment 109 
$7 \quad$ Bibliography

8 Appendix A 124

8.1 Business Process Management and Business Process Engineering 124

8.2 Service Oriented Architecture 125

$\begin{array}{lll}\text { 8.2.1 Enterprise Service Bus } & 128\end{array}$

$\begin{array}{ll}\text { 8.2.2 Web Services } & 129\end{array}$

$\begin{array}{lll}\text { 8.3 Workflow Management System } & 130\end{array}$

8.3.1 Workflow Components 132

8.3.2 Process Definition Language 134

8.3.3 Workflow Integration with other technologies 135

8.4 Scientific Workflow Management Systems 136

$\begin{array}{ll}\text { 8.4.1 Scientific Workflows Tools } & 137\end{array}$

$\begin{array}{ll}\text { 8.4.2 Condor } & 138\end{array}$

$\begin{array}{ll}\text { 8.4.3 InfoGrid } & 138\end{array}$

8.4.4 Grid Job Submission and Monitoring System 140

$\begin{array}{lll}8.5 & \text { Virtual Environments } & 140\end{array}$

9 Appendix B 143

$\begin{array}{lll}9.1 & \text { List of Publications } & 143\end{array}$ 


\section{List of Figures}

1.1 Collaboration of different simulations in an aircraft project. 20

1.2 Engineers in a collaborative section in oil \& gas. 21

1.3 Geologists, Geophysicists in a collaborative section 22

1.4 Deep-water production system layout. 23

2.1 DDMSF and its components. Architecture (left) and interaction scenario (right). $\quad 34$

2.2 Sequential workflow using customized wind tunnel grid activities. 36

2.3 The Vistrails Builder (left) and Vistrails Spreadsheet (right) 38

2.4 IDP Desktop interface and an IDP user interacting with the virtual world. 40

2.5 Multi-viewpoint image rendering. 41

2.6 Multiple users interacting with multiple workspaces. 42

2.7 A 3D fold surface calculated from the virtually mapped data (left). Isosurface showing aliasing in the simulation viscosity field (right). $\quad 43$

2.8 Automated monitoring system (left). RTAP web services layer (right). $\quad 44$

2.9 IAM graphical modeling tool (left) and its architecture (right). 45

3.1 CEE Conceptual Model. 51

3.2 Basic Service of the CEE SOA Architecture. 64

3.3 CEE Project Workflow. 66

$\begin{array}{ll}3.4 & \text { Overview of the user interaction with CEE. }\end{array}$

3.5 Creating the collaborative visualization session 68

3.6 Selecting the coordinator, the kind of session and the users that will $\begin{array}{ll}\text { participate. } & 68\end{array}$

3.7 Sending commands to load a simulation to visualize in the session 69

3.8 Riser simulation visualized in collaborative session. 69

$\begin{array}{ll}\text { 4.1 CEE Architecture Layers. } & 71\end{array}$

4.2 CEE Detailed SOA Architecture. 73

4.3 Registering available services in Service Registry. 80

4.4 Importing the Project Workflow from a ERP system. 81

4.5 Modeling and Executing a Scientific Workflow. 83

4.6 Executing a Scientific Workflow instance in CEE. 84

4.7 Starting a VC session in CEE. 85

$\begin{array}{ll}\text { 4.8 Environ and its RemoteCommandManager. } & 88\end{array}$ 
4.9 Starting a Visualization session in CEE. 90

5.1 Constructing the Riser Analysis workflow on BPEL Designer. 93

5.2 CEE SOA state of execution of a Riser Analysis Workflow. 94

5.3 Riser Analysis in CEE (Environ + CSV Tool). 95

5.4 Closer look on an element of the riser 97

5.5 Two users in a CEE collaborative visualization session. 98

$\begin{array}{ll}\text { 5.6 Measurments in a visualization session } & 99\end{array}$

5.7 Users monitoring the behavior of marine buoyant 100

5.8 Maintenance plan enriched with annotations. 102

5.9 Measurements in a CAD. 102

5.10 Engineering information. 103

5.11 Virtual Guided Tour 105

6.1 Towards tele-imersive CPSEs. 109

$\begin{array}{ll}7.1 \text { Business Process Engineering for CEE } & 125\end{array}$

7.2 Service-oriented terminology. (IBM RedBooks) 126

$\begin{array}{ll}7.3 \text { Collaboration in SOA (IBM RedBooks) } & 127\end{array}$

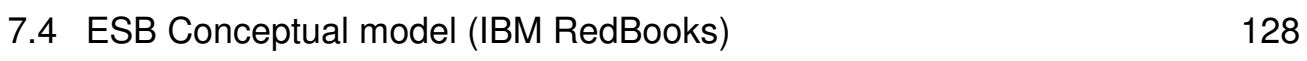

$\begin{array}{ll}7.5 \text { WfMC reference model. } & 133\end{array}$

7.6 Workflow pattern Sequence in XPDL. 134

$\begin{array}{ll}7.7 \text { WfMC reference model. } & 134\end{array}$

$\begin{array}{ll}7.8 & \text { InfoGrid architecture. } \\ & 139\end{array}$ 


\section{List of Tables}

2.1 Feature comparisons between CEE and related solutions 


\title{
Shortening
}

\author{
API - Application Program Interface \\ AWT - Abstract Windowing Toolkit \\ BPM - Business Process Management \\ CEE - Collaborative Engineering Environment \\ CFD - Computational Fluid Dynamics \\ CFF - Component Framework Framework \\ CLOS - Common Lisp Object System \\ CLX - Component Library for Cross Platform \\ CMS - Content Management System \\ COCA - Collaborative Objects Coordination Architecture \\ COM - Component Objetc Model \\ COPSE - Collaborative Project Support Environment \\ CORBA - Common Object Request Broker Architecture \\ CoWfMS - Collaborative Workflow Management Systems \\ CPSE - Collaborative Problem Solving Environment \\ CRIWG - International Workshop on Groupware \\ CSCA - Computer Supported Collaborative Argumentation \\ CSCW - Computer Supported Cooperative Work \\ CVEs - Collaborative Virtual Environments \\ DACIA - Dynamic Adjustment of Component InterActions \\ DISCIPLINE - DIstributed System for Collaborative Information Processing \\ and LEarning \\ DAO - Data Access Objects \\ DTO - Data Transfer Object \\ EJB - Enterprise Java Beans \\ EBR - First Seminar on Advanced Research in Electronic Business \\ ERP - Enterprise Resource Planning \\ FAQ - Frequently Asked Question \\ FPSO - Floating production, storage, and offloading production unit \\ FSO - Floating, storage, and offloading production unit \\ FTP - File Transfer Protocol \\ GC - Grid Computing \\ GPL - GNU General Public License
}




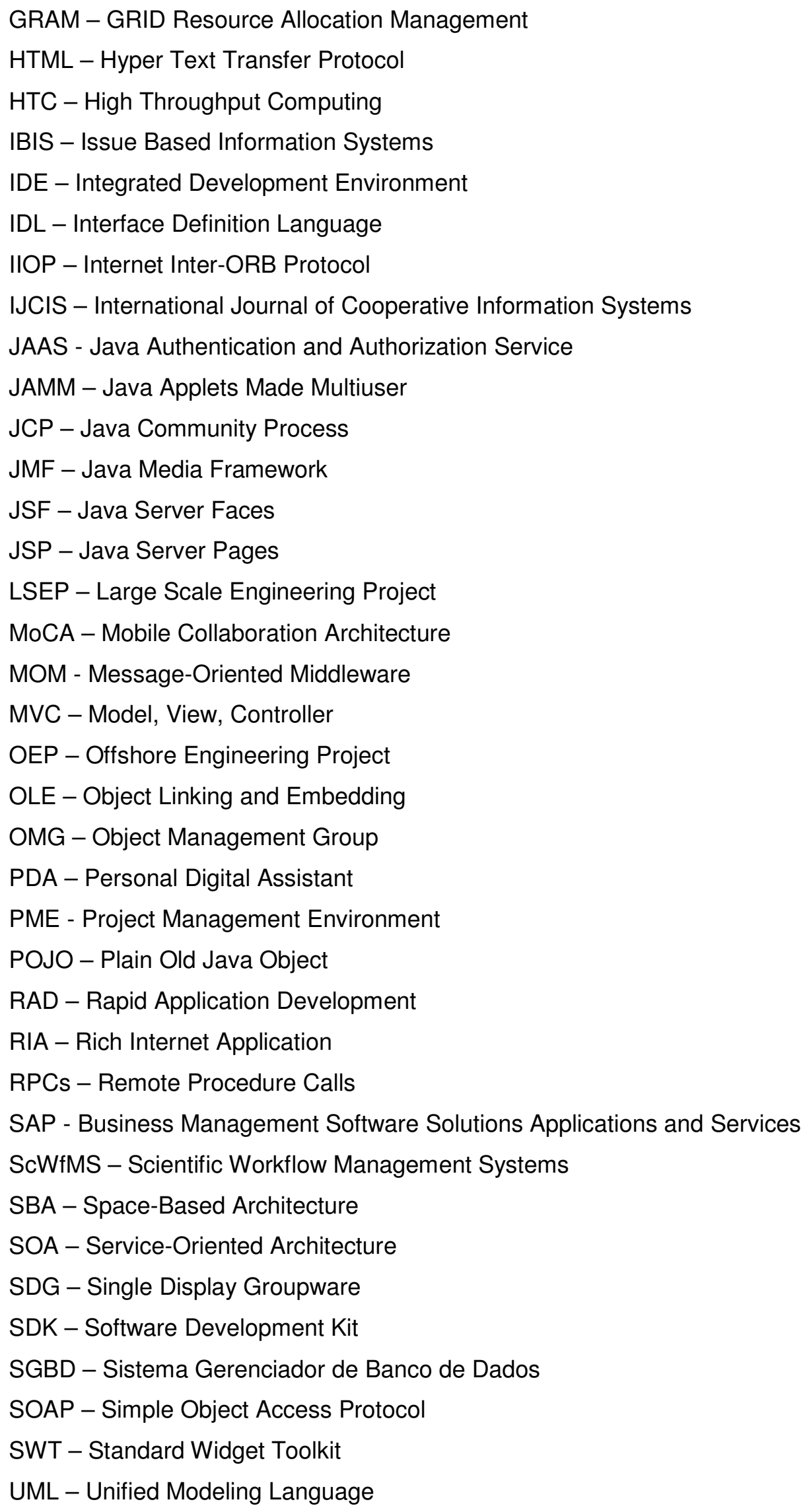




$$
\begin{aligned}
& \text { VC - Videoconference } \\
& \text { VCS - Videoconference System } \\
& \text { VNC - Virtual Networking Computing } \\
& \text { VE - Virtual Environments } \\
& \text { VR - Virtual Reality } \\
& \text { VRCs - Virtual Reality centers } \\
& \text { VRGeo - Virtual Reality for Geosciences } \\
& \text { VRML - Virtual Reality Modeling Language } \\
& \text { WYSIWIS - What You See Is What I See } \\
& \text { WfMC - Workflow Management Coalitio } \\
& \text { WfMS - Workflow Management Systems } \\
& \text { WYSIWYG - What You See Is What You Get } \\
& \text { XML - Extensible Markup Language } \\
& \text { XOOPS - eXtensible Object Oriented Portal System } \\
& \text { XSL - Extensible Style Language }
\end{aligned}
$$

